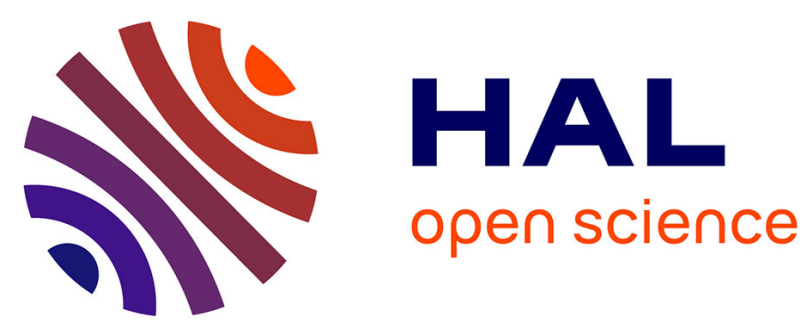

\title{
Optimal Observability-based Modelling, Design and Characterization of Piezoelectric Microactuators.
}

Roba El Khoury Moussa, Mathieu Grossard, Mehdi Boukallel, Arnaud Hubert, Nicolas Chaillet

\section{- To cite this version:}

Roba El Khoury Moussa, Mathieu Grossard, Mehdi Boukallel, Arnaud Hubert, Nicolas Chaillet. Optimal Observability-based Modelling, Design and Characterization of Piezoelectric Microactuators.. Smart Materials and Structures, 2013, 7, pp.1-13. 10.1088/0964-1726/22/7/075036 . hal-00839588

\section{HAL Id: hal-00839588 https://hal.science/hal-00839588}

Submitted on 28 Jun 2013

HAL is a multi-disciplinary open access archive for the deposit and dissemination of scientific research documents, whether they are published or not. The documents may come from teaching and research institutions in France or abroad, or from public or private research centers.
L'archive ouverte pluridisciplinaire HAL, est destinée au dépôt et à la diffusion de documents scientifiques de niveau recherche, publiés ou non, émanant des établissements d'enseignement et de recherche français ou étrangers, des laboratoires publics ou privés. 


\title{
Optimal Observability-based Modelling, Design and Characterization of Piezoelectric Microactuators
}

\author{
Roba El Khoury Moussa, Mathieu Grossard, Mehdi Boukallel, \\ Arnaud Hubert and Nicolas Chaillet
}

R. El Khoury Moussa and M. Grossard are with the Interactive Robotics Laboratory, LIST, CEA, F-92265 Fontenay aux Roses, France ;

M. Boukallel is with the Sensory and Ambient Interfaces Laboratory, LIST, CEA, F-92265 Fontenay aux Roses, France ;

A. Hubert and N. Chaillet are with the Automatic Control and Micro-Mechatronic Systems Department, FEMTO-ST Institute, Unite Mixte de Recherche CNRS 6174 -University of Franche-Comte (UFC)/Ecole Nationale Superieure de Mecanique et des Microtechniques (ENSMM)/University of Technology of Belfort-Montbeliard (UTBM), F-25000 Besancon, France.

E-mail: mathieu.grossard@cea.fr

\begin{abstract}
This paper deals with the optimal design of monolithic piezoelectric microactuators with integrated proprioceptive sensors. Dedicated to the microrobotic and micromechatronic fields, these works detail the modelling and the characterization of compliant structures with integrated actuating and sensing elements. The proposed optimal design procedure adresses not only static criteria but also dynamic ones. This leads to microdevices which are more performant with regards to mechanical (displacement, force...) and control (dynamics, stability, precision) characteristics. Efficient design of such devices is achieved using a flexible building block method. A topological optimization method combined with an evolutionary algorithm is used to optimize the design of truss-like planar structure. This method chooses the best location among the different piezoelectric elements. Different mechanical, actuation or sensing elements are accordingly chosen from a data bank. From the control point of view, optimisation criteria are considered to enforce the observability of the vibrational dominant modes of the structure. Therefore, control and observation Gramians are exploited in the optimal design to shape the open loop frequency response of both, actuation and sensing functions of the integrated device. In the last part of the paper, based on these results, the optimal design and manufacturing of an innovative piezoelectric flexible microgripper is proposed. The prototype is manufactured from a monolithic piezoelectric material (PIC 151). Its reduced size (15 mm x $18 \mathrm{~mm}$ ) fits the requirement of both microrobotics and micromechatronics applications, which is suitable for micromanipulation tasks. The characterization and the performance of this integrated microactuator finally close the paper and the efficiency of the optimal design procedure for micromechatronics applications is shown.
\end{abstract}




\section{Introduction}

In the micromanipulation and microrobotic field, microgripper devices use mainly compliant mechanisms, i.e. flexible monolythic structures undergoing elastic strain to transmit motion [1]. These compliant mechanisms replace favourably jointed rigid body motions with conventional articulated mechanisms. At the small scale (e.g. in microrobotics and micromechatronics), compliant mechanisms present many advantages: simplified manufacturing, reduced assembly costs, reduced kinematic and noise, no wear, no mechanical backlash, high precision, and ability to accommodate unconventional actuation schemes. The most interesting type of smart material used in such a matter is the piezoceramic PZT. Piezoelectric actuation has become widespread in micromanipulation systems where high positioning accuracy is required [2]. Beyond their appealing properties in the sense of micromechatronics design, an underlying advantage of piezoelectric materials is the reversibility of their electromechanical coupling effect, which explains their potential use in microrobotic applications as actuators and/or sensors.

The design of microrobotic structure with integrated active/smart multifunctional material is nevertheless a non-intuitive task (figure 1). The designer faces the problem of the physical integration of actuators and sensors in the compliant mechanism (i.e. the choice of the size, topology, material,...), as well as their optimal placement regarding the performances that can be achieved by a feedback or feedforward control of the integrated device [3].
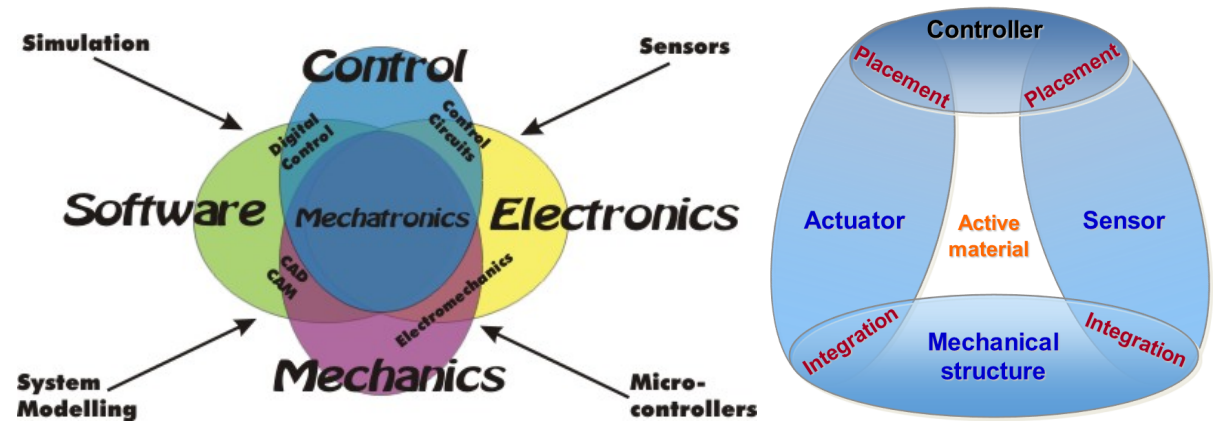

Figure 1. Mechatronics and smart structures design.

Numerous papers address the problem of optimal placement of piezoelectric actuator and sensor parts within flexible structures. The main field of interest for engineering applications concerns the active vibration control where minimization of undesired spillover effects is required $[4,5,6]$. Most works conducted on this subject are based on controllability and observability modal analysis of the compliant structure, performed separately for the optimal placement of actuator and sensor. Another related work concern the topology optimization which solves the problem of distributing a given amount of bulk material in a design domain subject to loading and support conditions. Design objectives, like stiffness or weight, have to be maximized or minimized. Various topol- 
ogy optimization methods have been applied to the design of piezoelectric devices [7, 8]. However, most of these methods consider only the optimal distribution of elastic passive materials while the locations of piezoelectric materials are already predefined. Indeed, very few studies consider the topology optimization (shape) of monolithic PZT active structures as reported in [9] or deals with the distribution of piezoelectric materials for the design of monolithic piezoelectric actuators or sensors. To our knowledge, no paper have been published previously concerning the topology optimization of monolithic piezoelectric devices that fully integrates actuation and sensing components. In addition, very few related works deal with topological optimization methods including frequency response analysis. The objective functions generaly aim at the maximization of either geometrical advantage (stroke amplification), or mechanical advantage (force amplification) in the static case [10]. Even if dynamic responses are considered in some papers, it is mostly in the restrictive case of predetermined harmonic loadings. As a consequence, it should be useful to optimize all these criteria from the first designing step, taking into account versatile microrobotic aspects to improve significantly the performances of such active compliant micromechanisms. A complete optimization process during the design steps would address several problems, such as avoiding noise amplification, decoupling disturbances, avoiding spillover-effects and improving dynamic input/output performances which can be of great interest in the robust control-oriented design of micromechatronic devices.

In this paper, we adress the optimal design of multifunctional piezoelectric compliant micromechanisms, which integrate actuation and sensing functions for microrobotics applications. Compared to existing optimization methods, our approach has the benefit of simultaneously combining the mechanical and the control design. Thus, both static and dynamic behaviours of the prototype are enhanced by the appropriate placement of the actuator and the sensing elements. Firstly the optimisation tools based on genetic algorithm is presented. This tool is specifically dedicated to compliant structures with piezoelectric materials. Based on previous works of authors taking into account the dynamic behaviour of the structure [11, 12, 13], we introduce a control-based criteria used during the optimal design process to increase the observability and the controllability of the system [14]. Such criteria facilitates the design of dynamic observer for position feedback control (estimation of the position based on direct piezoelectric effect). The resulting optimization method is then applied to design a fully integrated piezoelectric structure dedicated to micromanipulation purpose. Some details about the performances of the manufactured device finally close the paper.

\section{A compliant mechanisms stochastic design methodology}

In this section, we briefly present the flexible building block method, which has been implemented for the optimal design of micromechanical planar mechanisms in a software called FlexIn (developed with Matlab). Readers can find detailed descriptions of the 
method in $[11,12,15]$.

\subsection{General approach}

This optimization tool is based on a multiobjective genetic algorithm approach, which searchs the optimal distribution of available building blocks. The resulting structure is thus an assembly of elementary passive, active or sensitive compliant blocks chosen in a library (figure 2). Some blocks can act as pure mechanical stiffness, whereas others integrate actuation functions and others sensing functions. The optimization problem appoints not only topological specifications, but also an optimal set of boundary conditions (fixed frame location, contacts, end-effectors, etc), dimensions and materials, depending on some optimization criteria selected by the designer.

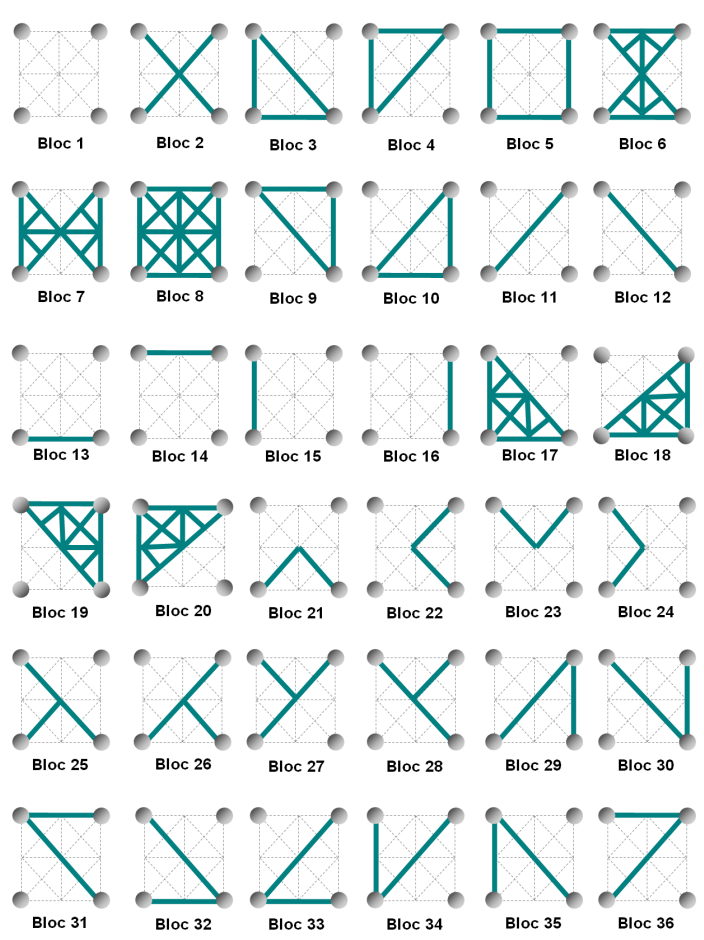

Figure 2. Library of compliant passive, active or sensitive piezoelectric building blocks for planar compliant mechanisms synthesis using FlexIn.

\subsection{Electro-mechanical FE model of the piezoelectric sensing structures}

The modelling of active and sensitive piezoelectric building blocks is based on a 2D Finite Element Method (FEM) as presented in this section.

2.2.1. FE formulation of the piezoelectric beam In the optimization procedure, the computation of different criteria requires the FE model of each block of the library. To obtain the FE formulation of the piezoelectric blocks, a model of a piezoelectric beam exploites direct and inverse piezoelectricity effects for sensing and actuation purposes 
respectively (figure 3). In the current version of FlexIn, a block is used either as sensor or as actuator but not for the two functions simultaneously. Nevertheless, it could change in future version of FlexIn.

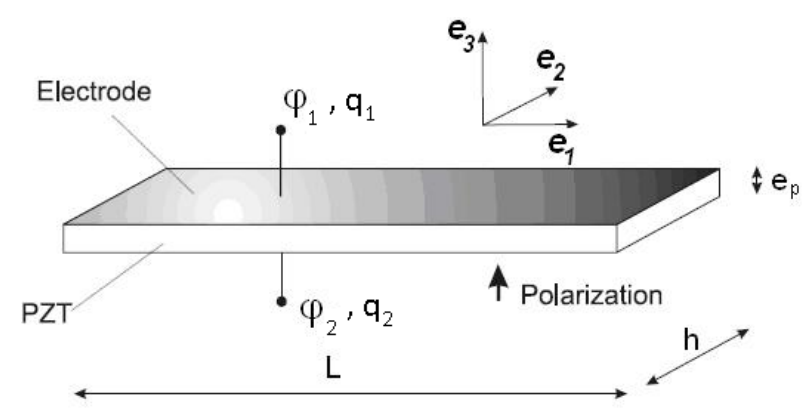

Figure 3. Thickness-polarized piezoelectric beam transducer with electroded surfaces, and orientation in the material reference frame $\left(\mathbf{e}_{\mathbf{1}}, \mathbf{e}_{\mathbf{2}}, \mathbf{e}_{\mathbf{3}}\right) .\left(\varphi_{\mathbf{1}}, \varphi_{\mathbf{2}}\right)$ and $\left(q_{1}, q_{2}\right)$ denote respectively the electric potential for actuation case, and the electric charges for sensing case, of the two electrodes.

The matrices characterising each block (mass, stiffness, electromechanical coupling and electric capacity) are computed at the beginning of the optimization algorithm, by the association of the corresponding beam matrices in the global coordinate system of the structure. The compliant mechanism is assumed to undergo structural planar deformation, mainly due to flexion, leading to considering Navier-Bernoulli beams type FEs. Exploiting the transverse effect of piezoelectricity, longitudinal deformation along $L$ dimension is generated under the transverse electric field along $e_{p}$ thickness. Using least action Hamilton principle for electromechanical system [16], piezoelectric beam model is established as a set of two equations, one for the actuation mode (inverse effect) and one for the sensing mode of working (direct effect):

$$
\begin{aligned}
& \mathbf{M}_{\mathbf{b}} \ddot{\eta}_{\mathbf{b}}+\mathbf{K}_{\mathbf{b}} \eta_{\mathbf{b}}=\mathbf{G}_{\mathbf{b}} \boldsymbol{\Phi}_{\mathbf{b}}+\mathbf{F r}_{\mathbf{b}} \\
& \mathbf{q}_{\mathbf{b}}=\mathbf{G}_{\mathbf{b}}{ }^{t} \eta_{\mathbf{b}}+\mathbf{C}_{\mathbf{b}}{ }^{t} \boldsymbol{\Phi}_{\mathbf{b}}
\end{aligned}
$$

$\eta_{\mathbf{b}}=\left(u_{A}, v_{A}, \omega_{A}, u_{B}, v_{B}, \omega_{B}\right)_{R_{p}}^{t}$ is the nodal displacement vector in the beam's coordinate system $R_{p}=\left(A, \mathbf{x}_{\mathbf{p}}, \mathbf{y}_{\mathbf{p}}, \mathbf{z}_{\mathbf{p}}\right)$ (figure 4$) . \mathbf{M}_{\mathbf{b}}, \mathbf{K}_{\mathbf{b}}, \mathbf{G}_{\mathbf{b}}$ and $\mathbf{C}_{\mathbf{b}}$ are respectively the mass, stiffness, electromechanical coupling and electric capacitive beam matrices. Corresponding matrices are given in the Appendix section. $\boldsymbol{\Phi}_{\mathbf{b}}=\left[\varphi_{1}, \varphi_{2}\right]^{t}$ and $\mathbf{q}_{\mathbf{b}}=\left[q_{1}, q_{2}\right]^{t}$ are respectively the vectors of electric potentials and electric charges on the upper and lower boundaries of the piezoelectric beam.

Matrix $\mathbf{G}_{\mathbf{b}}$ in (1) induces piezoelectric loads, which makes the actuator beam expand (or contract) proportionally to the external applied voltage $\varphi_{1}-\varphi_{2}$. The forces vector $\mathbf{F r}_{\mathbf{b}}$, is due to external mechanical loads at the extremities of the beam

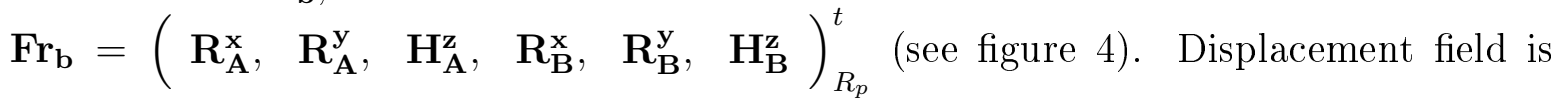
related to the corresponding node displacement $\eta_{\mathbf{b}}$ by the mean of the shape functions, computed according to Navier-Bernoulli kinematic assumptions. The relationship (2) is used to compute the electric charges induced on the sensing beam electrodes according 


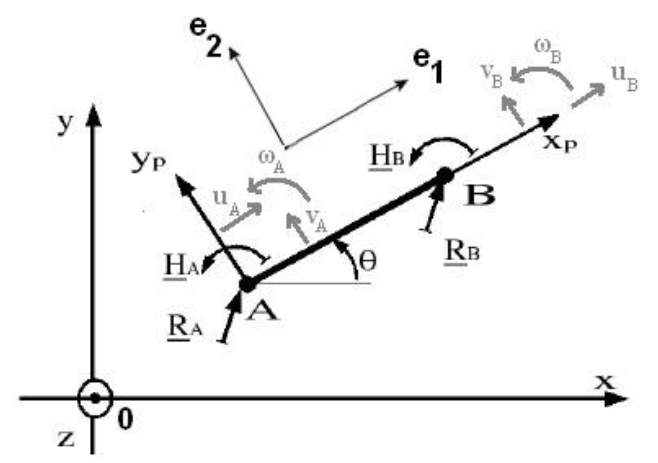

Figure 4. Curvilinear coordinates of the piezoelectric beam $A-B$, and its orientation in the global coordinate system $R^{\prime}=(0, \mathbf{x}, \mathbf{y}, \mathbf{z}) . \mathbf{R}$ and $\mathbf{H}$ represent the in-plane nodal force and moment at the beam extremities.

to the direct effect of piezoelectricity. It must be noticed that experimental measurement of electric charges requires an electronic charge-voltage converter using operational amplifiers. In ideal mode, upper and lower electrodes of the sensors piezoelectric beam are then considered short-circuited as it would be the case when they will be connected to operational amplifier. A zero-voltage difference $\varphi_{1}-\varphi_{2}$ can therefore be assumed in the following and relationship (2) becomes $\mathbf{q}_{\mathbf{b}}=\mathbf{G}_{\mathbf{b}}{ }^{t} \eta_{\mathbf{b}}$ for sensing blocks. This assumption ensures that sensing and actuation functions of piezoelectric beams are decoupled.

2.2.2. FE model of the FlexIn piezoelectric structures The mass, stiffness, electromechanical coupling and electric capacity matrices of each block are computed, considering every combination of the discrete values allowed for the structural optimization variables, i.e. material and size of the blocks. Each block results from the association of the corresponding beam matrices in the global coordinate system of the structure. Thus, the calculation of the different matrices of each valued block is done one time only at the beginning of the optimal design problem (before running the genetic algorithm), which saves computing time.

Each flexible structure synthesized using blocks by FlexIn, is defined as a finitedimensional linear system modeled as:

$$
\left\{\begin{array}{l}
\mathbf{M}_{\mathbf{g}} \ddot{\eta}_{\mathrm{g}}+\mathbf{K}_{\mathrm{g}} \eta_{\mathrm{g}}=\mathbf{E}_{\mathrm{g}} \mathbf{u} \\
\mathbf{y}_{\mathbf{c o}}=\delta=\mathbf{F}_{\mathbf{g}} \eta_{\mathrm{g}} \\
\mathbf{y}_{\mathrm{ob}}=\mathbf{q}=\mathbf{L}_{\mathrm{g}} \eta_{\mathrm{g}}
\end{array}\right.
$$

The foregoing second-order differential matrix equations represent the undamped dynamic behavior of the device. $\mathbf{M}_{\mathbf{g}}$ and $\mathbf{K}_{\mathbf{g}}$ are respectively the structural mass and stiffness matrices, arising from the assembly of all blocks matrices which constitute the general structure.

Considering the integers $p, s$, and $r$, as respectively the numbers of DOFs of the structure, number of inputs (i.e. actuators), and number of tip displacement outputs, $\eta_{\mathrm{g}}$ is then the $p \times 1$ nodal displacement vector and $\mathbf{u}$ is the $s \times 1$ input vector. The 
$p \times s$ input matrix $\mathbf{E}_{\mathbf{g}}$ reflects the location of the actuated DOFs, while $\mathbf{y}_{\text {co }}$ is the $r \times 1$ controlled output vector representing the output tip displacement $\delta$ through the output displacement $(r \times p)$-matrix $\mathbf{F}_{\mathbf{g}}$. The third equation expresses the electric charges $\left(\mathbf{y}_{\mathbf{o b}}=\mathbf{q}\right)$ obtained by the integrated sensing function from the direct piezoelectric effect. Note that $\mathbf{L}_{\mathbf{g}}$ is the $1 \times p$ single output matrix pointing out the placement of piezoelectric sensor in the structure. Hence, it is important to note that there are two kinds of output: controlled output variable $\mathbf{y}_{\text {co }}$ and observed output variables $\mathbf{y}_{\text {ob }}$ that are different variables. This point does not correspond to the usual case of collocated control (output is the observed variable).

\section{A new performance criterion for proprioceptive observation}

To successfully achieve suitable dynamic open-loop performances, an optimal topology design strategy is derived taking into account control-observability criterion. This one, based on modal balanced and observability gramians matrix interpretations, will optimally place and integrate actuators and sensors in the microstructure. The physical coordinates $\eta_{\mathbf{g}}$ of (3) are firstly transformed into modal coordinates $\mathbf{z}$ to emphasize the flexible modes contribution. Then, taking advantage of the numerical simple expressions of the Hankel singular values in modal coordinates, a new criterion is proposed to optimize the topological of the structure.

\subsection{Modal Representation for flexible structures}

The harmonic solutions of the first equation in (3) give the eigenvectors matrix $\Psi$ and natural frequencies $\omega_{i}$ of the system. Details of the modal representation computation can be found in [12]:

$$
\left\{\begin{array}{l}
\ddot{\mathbf{z}}+\operatorname{diag}\left(2 \xi_{\mathrm{i}} \omega_{i}\right) \dot{\mathbf{z}}+\operatorname{diag}\left(\omega_{i}^{2}\right) \mathbf{z}=\Psi^{t} \mathbf{E}_{\mathbf{g}} \mathbf{u} \\
\mathbf{y}_{\mathbf{c o}}=\delta=\mathbf{F}_{\mathbf{g}} \Psi_{\mathbf{z}} \\
\mathbf{y}_{\mathbf{o b}}=\mathbf{q}=\mathbf{L}_{\mathbf{g}} \Psi_{\mathbf{z}}
\end{array}\right.
$$

where $\mathbf{z}$ is the $p \times 1$ modal displacement vector, and $\xi_{i}$ is the $i^{t h}$ modal damping ratio introduced using Basil's hypothesis. For a $p$-size degrees of freedom mechanical system, a $2 \times p$-size state vector must be defined. A typically $2 p \times 1$ state vector $\mathbf{x}$ (see [17] for advantages of this choice for flexible structures), consists of modal velocities and frequency-weighted modal displacements:

$$
\mathbf{x}=\left(\begin{array}{ccccc}
\dot{z}_{1} & \omega_{1} z_{1} & \ldots & \dot{z}_{p} & \omega_{p} z_{p}
\end{array}\right)^{t}
$$

Since controlled and observed output vectors are not the same, the modal statespace representation can be written as follows:

$$
\left\{\begin{array}{c}
\dot{\mathrm{x}}=\mathrm{Ax}+\mathrm{Bu} \\
\mathrm{y}_{\mathrm{co}}=\mathrm{C}_{\mathrm{co}} \mathrm{x} \\
\mathrm{y}_{\mathrm{ob}}=\mathrm{C}_{\mathrm{ob}} \mathrm{x}
\end{array}\right.
$$


which leads to two matrices triplets $\left(\mathbf{A}, \mathbf{B}, \mathbf{C}_{\mathbf{c o}}\right)$ and $\left(\mathbf{A}, \mathbf{B}, \mathbf{C}_{\mathbf{o b}}\right)$ related to control and observation state space models. The matrices take the forms $\mathbf{A}=\operatorname{diag}\left(\mathbf{A}_{\mathbf{1}}, \ldots \mathbf{A}_{\mathbf{p}}\right)$, $\mathbf{B}=\left(\mathbf{B}_{1}^{t}, \ldots \mathbf{B}_{\mathbf{p}}^{t}\right)^{t}, \mathbf{C}_{\mathbf{c o}}=\left(\mathbf{C}_{\mathbf{c o}_{1}}, \ldots \mathbf{C}_{\mathbf{c o}_{\mathbf{p}}}\right)$, and $\mathbf{C}_{\mathbf{o b}}=\left(\mathbf{C}_{\mathbf{o b}_{1}}, \ldots \mathbf{C}_{\mathbf{o b}_{\mathbf{p}}}\right)$, with, for $i=1, \ldots, p$,

$$
\begin{gathered}
\mathbf{A}_{\mathbf{i}}=\left[\begin{array}{cc}
-2 \zeta_{i} \omega_{i} & -\omega_{i} \\
\omega_{i} & 0
\end{array}\right] \\
\mathbf{B}_{\mathbf{i}}=\left[\begin{array}{c}
\mathbf{b}_{\mathbf{i}} \\
\mathbf{0}
\end{array}\right] \\
\mathbf{C}_{\mathbf{c o}_{\mathbf{i}}}=\left[\begin{array}{ll}
\mathbf{0} & \frac{\mathbf{c}_{\mathbf{c o}_{\mathbf{i}}}}{\omega_{i}}
\end{array}\right] \\
\mathbf{C}_{\mathbf{o b}_{\mathbf{i}}}=\left[\begin{array}{ll}
\mathbf{0} & \frac{\mathbf{c}_{\mathbf{o b}_{\mathbf{i}}}}{\omega_{i}}
\end{array}\right]
\end{gathered}
$$

$\mathbf{b}_{\mathbf{i}}, \mathbf{c}_{\mathbf{c o}_{\mathbf{i}}}$, and $\mathbf{c}_{\mathbf{o b}}$ are the $i^{\text {th }}$ components of $\boldsymbol{\Psi}^{t} \mathbf{E}_{\mathbf{g}}, \mathbf{F}_{\mathbf{g}} \Psi$ and $\mathbf{L}_{\mathbf{g}} \Psi$ respectively. Matrix $\mathbf{A}$ is a function of the structural parameters (eigen frequencies and damping ratio), whereas matrix $\mathbf{B}$ depends only on the location of actuated DOF, matrix $\mathbf{C}_{\mathbf{c o}}$ on the location of desired displacement output, and matrix $\mathbf{C}_{\mathbf{o b}}$ on the location of integrated piezoelectric sensors.

\subsection{Computation of the observability and balanced gramians matrices}

Observability gramian $\left(\mathbf{W}_{\mathbf{o b}}\right)$ between state $\mathbf{x}$ and measured output $\mathbf{q}$ is found to be convenient to characterize the modes observability by the mean of the measured electric charge q. Its energetic and geometric interpretations are emphasized in [4] and [18]. For stable A matrix, $\mathbf{W}_{\mathbf{~ o b}}$ is the algebraic solution of the following Lyapunov equation:

$$
\mathbf{A}^{t} \mathbf{W}_{\mathbf{o b}}+\mathbf{W}_{\mathrm{ob}} \mathbf{A}+\mathbf{C}_{\mathrm{ob}}^{t} \mathbf{C}_{\mathrm{ob}}=\mathbf{0}
$$

Assuming that the damping ratios are infinitely small and the natural frequencies well spaced, which is widely accepted for flexible structures, the block diagonal forms of $\left(\mathbf{A}, \mathbf{C}_{\mathbf{o b}}\right)$ couple can be exploited to give closed-form analytical expression of the modal observability gramian matrix [19]. The solution is then diagonal and equal to:

$$
\mathbf{W}_{\mathrm{ob}}=\operatorname{diag}\left(\mathbf{W}_{\mathrm{ob}_{11}}, \ldots, \mathbf{W}_{\mathrm{ob}_{\mathrm{pp}}}\right)
$$

with, for $i=1, \ldots, p$,

$$
\mathbf{W}_{\mathbf{o b}_{\mathbf{i i}}}=\frac{\gamma_{q_{i i}}}{4 \xi_{i} \omega_{i}^{3}} \mathbf{I}_{\mathbf{2}}=\alpha_{i} \mathbf{I}_{\mathbf{2}}
$$

where $\gamma_{q_{i i}}=\mathbf{c}_{\mathbf{o b}_{\mathbf{i}}}^{t} \mathbf{c}_{\mathbf{o b}_{\mathbf{i}}}$, and $\mathbf{I}_{\mathbf{2}}$ is the $2 \times 2$ identity matrix. For a given mode $\left(\xi_{i}, \omega_{i}\right)$, $\gamma_{q_{i i}}$ scalars represent the way the $i^{t h}$ mode is seen through the piezoelectric sensor blocks.

The dominant vibrational modes contributions, which govern the dynamics of the tip deflection, have to be fully observable from the integrated sensing area of the device, in order to efficiently reconstruct and control the tip deflection. This will give the kind 
of transfer function sketched on the figure 5: few dominant modes (high peaks) at low frequency in the transfer function between the tips position $\delta$ and the voltage input control $u$. Moreover, these few dominant modes have to be strongly coupled - located at the same frequencies - with the dominant modes of the transfer function between the electric charge $q$ in the sensing areas and the voltage input control $u$.

These dominant modes are symbolized by high Hankel Singular Values (HSVs), which defines the balanced gramian matrix $\mathbf{W}_{\mathbf{e} \delta}$ of $\left(\mathbf{A}, \mathbf{B}, \mathbf{C}_{\mathbf{c o}}\right)$ system as follows:

$$
\mathbf{W}_{\mathbf{c} \delta}=\mathbf{W}_{\mathbf{o} \delta}=\mathbf{W}_{\mathbf{e} \delta}=\operatorname{diag}\left(\sigma_{i}\right)
$$

where $\sigma_{\mathbf{i}}$ are the HSVs of the $\left(\mathbf{A}, \mathbf{B}, \mathbf{C}_{\mathbf{c o}}\right)$ system. Balanced gramian is therefore a useful tool to quantify the joint controllability and observability of the system. When damping ratios decrease to zero, it can be shown that modal state coordinates are approximately balanced, and the approximate $i^{\text {th }}$ HSV for flexible structure in this asymptotic situation is given by:

$$
\sigma_{i}=\frac{\sqrt{\mathbf{c}_{\mathbf{c o}_{\mathbf{i}}^{t}}^{t} \mathbf{c}_{\mathbf{c o}_{\mathbf{i}}} \mathbf{b}_{\mathbf{i}} \mathbf{b}_{\mathbf{i}}^{t}}}{4 \xi_{i} \omega_{i}^{2}}
$$

HSV describes the degree of the corresponding modal state's input-output energy flow through the system [19].

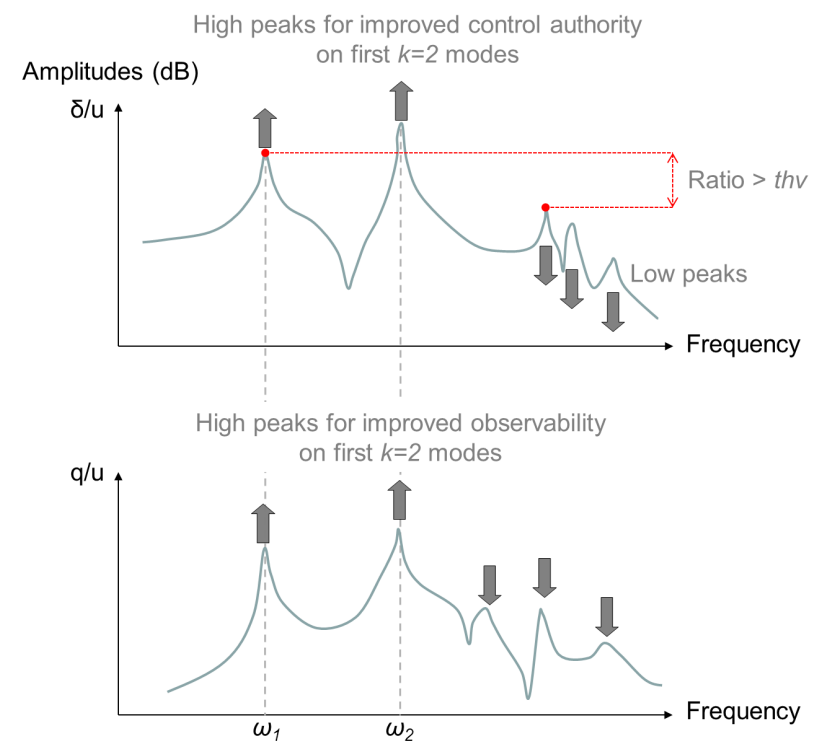

Figure 5. Example of desired control and observation transfers for good obeservability and controllability of the structure.

3.3. Gramian-based criterion for the optimization of the actuator and sensor integration within a piezoelectric micromechanism

As emphasized previously, the design case of active structures with both integrated actuation and sensing capabilities is non-inuitive and the designer is faced with two main issues: 
(i) A reduced model of the structure must be synthesized, which includes the few dominant low-frequency modes, without destabilizing the system by rejecting the residual modes (i.e. high roll-off after the dominant modes as sketched in the figure 5). Resonance peaks amplitudes must be maximized in the frequencies bandwidth to increase authority control on the dominant modes of the deflection transfer. On the contrary, the amplitudes of resonance peaks after cut-off frequency must be minimized to increase gain margin and to limit modes destabilization in this area (spillover phenomenon).

(ii) If the dominant modes are not all well observed by the sensing area of the flexible structure, the reconstruction of the deflection $\delta$ will not be guaranteed in an optimal way by the observer.

Hence, we propose a new criterion which solves these issues: Forcing the optimal structure to have $k$ first dominant modes in the $\delta / \mathbf{u}$ frequency response function solves the first point. Guaranteeing a high-level of observability of these dominant modes by means of the electric charges $\mathbf{q}$ solves the second point.The algorithm 1 express this procedure.

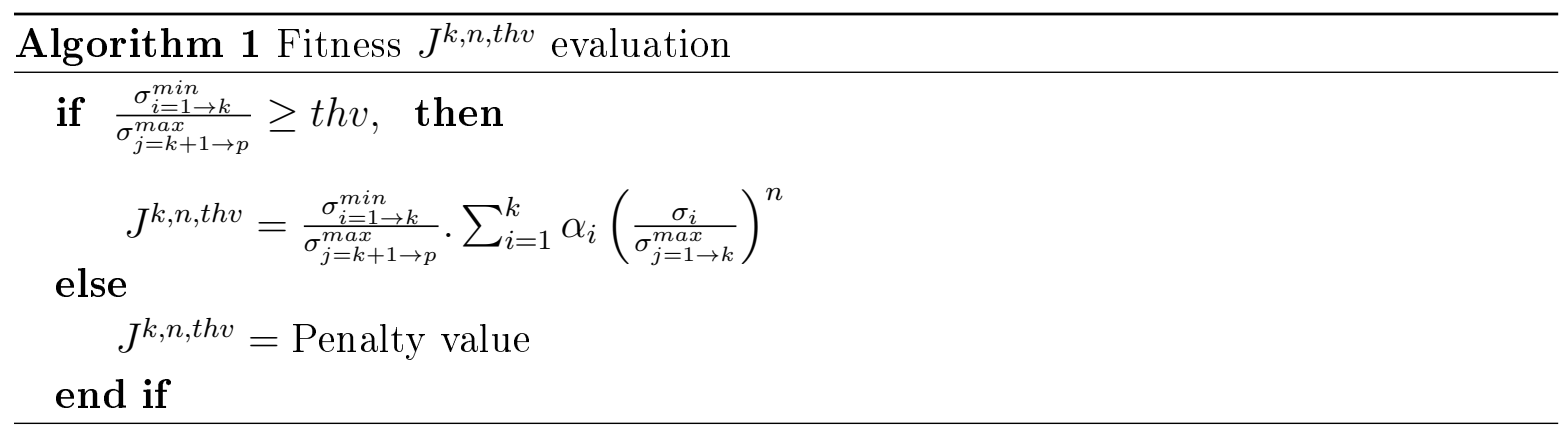

In this algorithm, $k$ is the number of the first dominant modes specified by the designer. $\sigma_{i=1 \rightarrow k}^{\min }$ and $\sigma_{j=k+1 \rightarrow p}^{\max }$ are respectively the level of the least dominant modes belonging to the first $k$ modes and the level of the most dominant modes belonging to the residual modes. $t h v$ is a threshold value specified by the designer. As can be seen in the figure 5 , the condition $\frac{\sigma_{i=1 \rightarrow k}^{\min }}{\sigma_{j=k+1 \rightarrow p}^{m a x}} \geq t h v$, means that the $k$ first modes dominate by at least thv times the residual modes. $\alpha_{i}=\frac{\gamma_{q_{i i}}}{4 \xi_{i} \omega_{i}^{3}}$ corresponds to the coefficient of the $i^{t h}$ observability modal gramian $\mathbf{W}_{\mathbf{o b}_{\mathbf{i i}}}$ and $\left(\sigma_{i} / \sigma_{j}^{\max }\right)^{n}$ is a weighing ratio $\in[0,1]$. When this ratio is close to 1 , the corresponding $i^{t h}$ mode is a dominant mode belonging to the first $k$ modes. The exponent $n$ is a tuning parameter to accelerate the convergence towards $\sigma_{i}^{\max }$ (to emphasize the most dominant modes). Thus, the corresponding $\alpha_{i}$ is privileged compared to other modes observability. Maximizing this criterion favors flexible structures with vibrational modes where good observability of $\delta$ coincides with its dominant modes. 


\section{Application of the method to the design of piezoelectric mechanisms}

The previous section presented a new multiobjective optimal method for the design of smart compliant structure. In this section, this method is applied to the synthesis of a monolithic micromechanism with integrated piezoelectric actuator and sensor.

\subsection{Optimization problem specifications}

The monolithic structure is seen as the actuator of a left-arm gripper and is supposed to be made of a single piezoelectric material PIC151 from PI Piezo Ceramic Technology [20]. The structure topology is considered to have a maximal size of $15 \mathrm{~mm} \times 18$ $\mathrm{mm}$, and a constant thickness of $200 \mu \mathrm{m}$. This device includes either passive, active or sensitive blocks inside a $2 \times 3$ mesh (see figure 6 ). The number of active (resp. sensitive) blocks in the structure can vary between 1 and 4 . These ones are optimally chosen in the library displayed in the figure 2 . When external voltages are applied to the blocks electrodes, the output node (tip of the structure) has to move along the $x$-axis. To evaluate static mechanical criteria, the electric voltage value applied between upper and lower electrods of active blocks is $200 \mathrm{~V}$. The size ratio of the blocks can vary as $b_{\max } / b_{\min } \in \llbracket 1 ; 2 \rrbracket$ and $a_{\max } / a_{\min } \in \llbracket 1 ; 2 \rrbracket$ ( $a$ and $b$ geometric parameters are displayed in the figure 6). The number of blocked nodes is comprised between 1 and 3 among the locations permitted by the optimization problem.

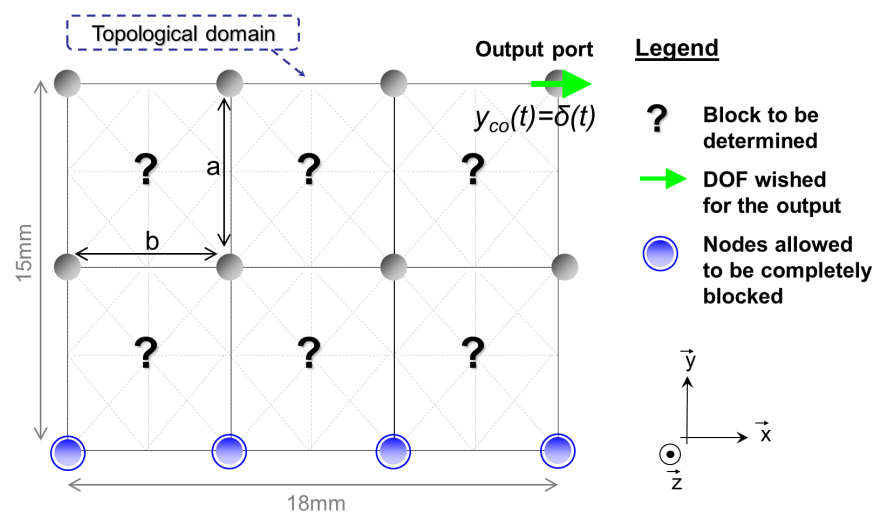

Figure 6. Mesh of the PZT compliant micromechanism with imposed and allowed boundary conditions. $a$ and $b$ optimization parameters define the relative height and width of the blocks.

Finally, three numerical criteria to be maximized with FlexIn are considered :

- $J^{1}$ : free mechanical displacement $\delta_{\mathbf{x}}$ at the output node in x-direction when a constant voltage is applied to the system,

- $J^{2}$ : amplitude of the sensing electric charges induced on the sensing piezoelectric blocks,

- $J^{k, n, t h v}$ : modal observability of the mechanism output $\delta$ by the observed sensitive blocks charges $\mathbf{q}$. In this example, we chose $k=2, n=2$ and $t h v=3$. 
Note that $J^{1}$ and $J^{2}$ consider only the static behavior of the structure, while $J^{k, n, t h v}$ considers its dynamic behavior.

\subsection{Results and Validation}

The optimization procedure was started according to the previous specifications. When the genetic algorithm does not find any new pseudo-optimum during 200 subsequent generations, the best compromises are kept and can be found on Pareto fronts. The designer can chose among these solutions, after studying their static and dynamic behaviors. In our case, the structure chosen appears to have the best dynamic desired behavior according to $J^{3}$, while static criteria remain relatively good. This structure, displayed in the figure 7, allows a tip displacement of $7 \mu \mathrm{m}$, and induces sensing charges around $1.9 \cdot 10^{-9} \mathrm{C}$ under a $200 \mathrm{~V}$ input voltage.

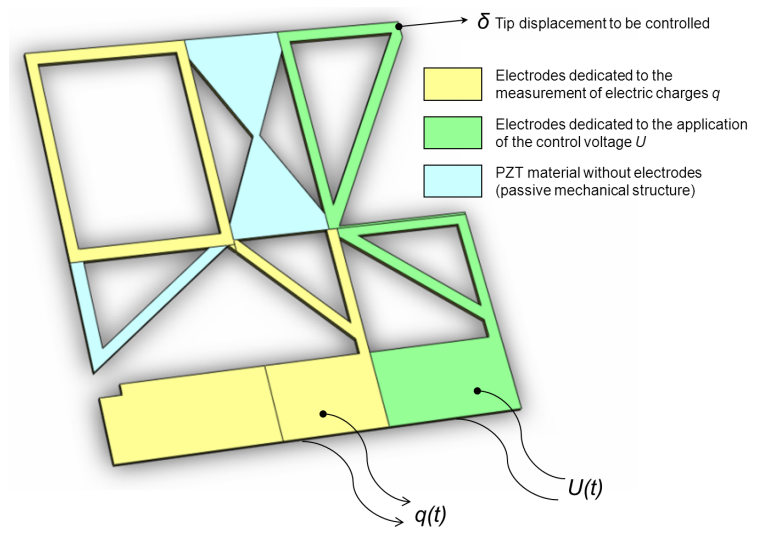

Figure 7. 3D CAD of the left-finger microactuator integrating sensing capabilities.

The figure 8 reports the simulated dynamic behaviour (Bode diagrams) of $\delta / \mathbf{u}$ control transfer function and $\mathbf{q} / \mathbf{u}$ observation transfer function. According to design specifications, theses results show that maximizing the $J^{2,2,3}$ criteria has led to the occurrence of two dominant resonant modes in the low-frequency spectrum of the deflection response, and these latter are perfectly observed by the electric charges.

\section{Characterization and identification of the designed structure}

In this section, we characterize the complet electromechanical model of the tip deflection of the manufactured piezoelectric device as a function of the input control voltage. The model is then identified based on experimental measurements.

\subsection{Experimental setup}

The microsystem prototype is clamped, and placed on $x-y-z$ micropositioning linear stages, which are manually operated. The figure 9 shows a photograph of the experimental setup. The piezoelectric actuator requires high voltage (about $\pm 100 \mathrm{~V}$ ) 


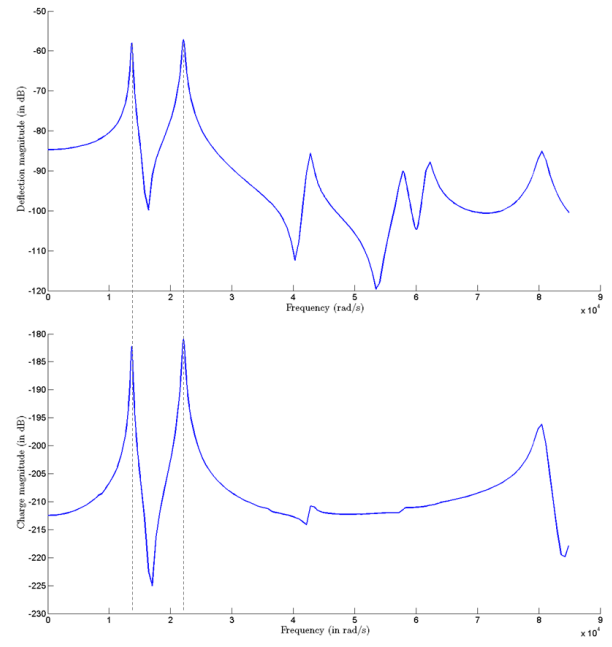

Figure 8. Bode diagrams of control and observation transfers of the selected structure.

to provide micrometric deflection. Thus, the device is supplied with a linear power amplifier (amplification ratio $=50$ ). As seen in the figure 10, the device is computercontrolled using Matlab-Simulink software and a NI Labview PXI board (sampling frequency $f_{e}=20 \mathrm{kHz}$ ). Output displacement of the tip of the piezoelectric structure is directly measured along $x$-axis using a $0.01 \mu$ m-resolution Keyence laser sensor.

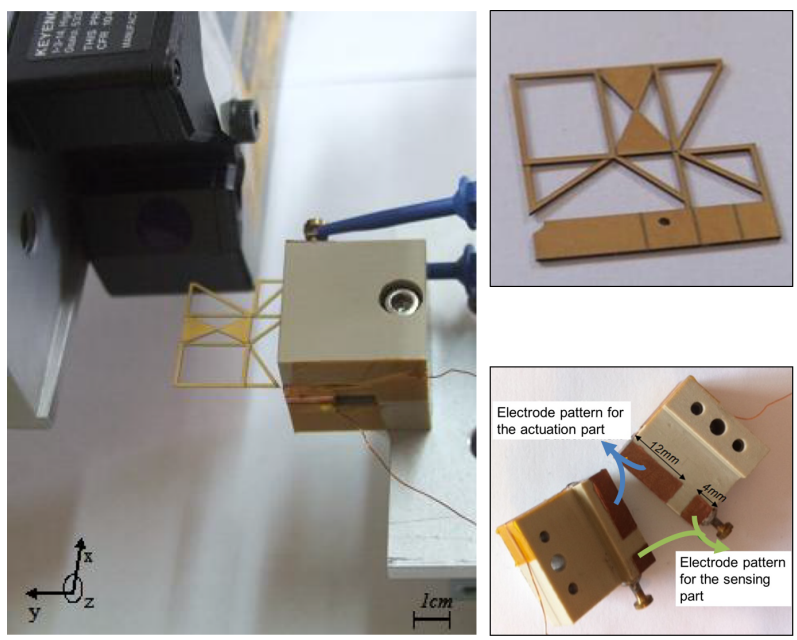

Figure 9. Experimental setup: manufactured piezoelectric monolithic device (laser cutted electroded PIC151 plate), clamping system and electrode patterns for the actuation and sensing areas.

An electronic charge-voltage converter is used to measure the electric charges induced in the sensing areas. The analog outputs of laser sensor and charge amplifiers are directly wired to a $4^{\text {th }}$-order low-pass anti-aliasing filter. Corresponding double SallenKey electronic circuits are tuned to provide more than $75 \mathrm{~dB}$ attenuation at the $\frac{f_{e}}{2}=10$ $\mathrm{kHz}$ Shannon frequency. This permits to filter accurently high frequencies noise and high frequencies unmodelled dynamics. 


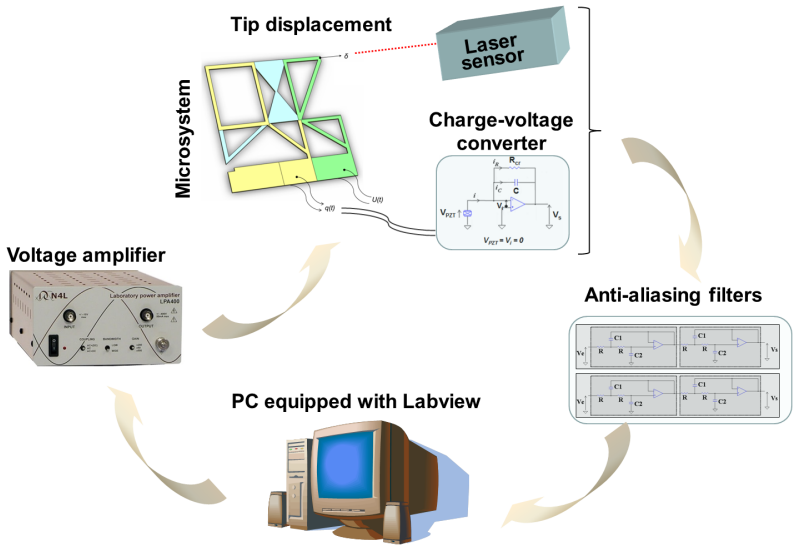

Figure 10. Hardware description of the computer-controlled setup.

In ideal operating mode, upper and lower electrodes of the sensing piezoelectric beams are considered short-circuited to respect the optimization design hypothesis. This assumption ensures that sensing piezoelectric beams are only related to the local deformation of the microactuator. The electronic signal conditioner is based on an operational amplifier in a current integrator operating mode (see figure 11). This analog conditioner for the charges measurement on the sensing electrodes pattern uses a resistor $R_{c r}$ in parallel to the feedback capacitance $C$. The resistor's role is to discharge the capacitance $C$ to avoid saturation effects at low frequencies. This ensures the proper functioning of the electric charges integration. The measured output voltage $V_{s}$ is related to the electric charges $q$ in s-domain as follows:

$$
V_{s}(s)=-\frac{1}{C}\left(\frac{s}{s+\frac{1}{R C}}\right) Q(s) .
$$

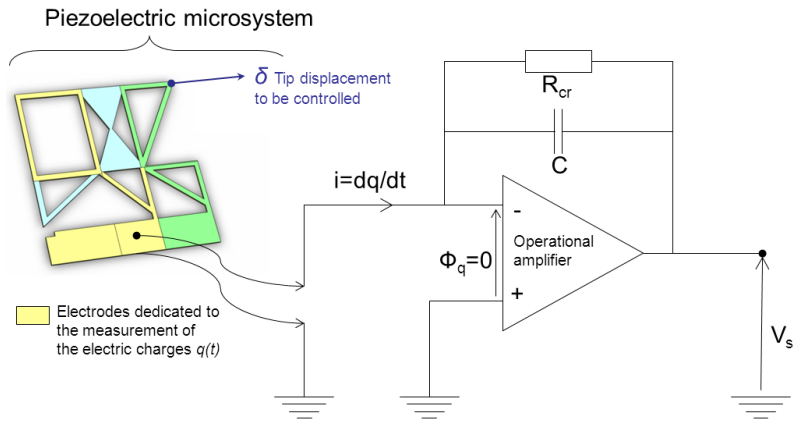

Figure 11. Electronic circuitry for the measurement of the electric charges $q(t)$ based on charge-voltage converter.

Micropower precision operational amplifiers ( $L F 412 A C N$, National Semiconductor Corporation) are used because they present a very low voltage drift with respect to the temperature $\left(\max . \pm 0.4 \mu \mathrm{V} /{ }^{\circ} \mathrm{C}\right)$, a small offset voltage $(\max .= \pm 150 \mu \mathrm{V} \max )$. Its 
performances in terms of stability and accuracy and its high bandwidth $(130 \mathrm{kHz})$ make it suitable for signal conditioner. In this case, it is able to measure $\mathrm{nC}$ electric charges. The $C$ feedback capacity is chosen equal to $2.2 \mathrm{nF}$, a value close to the experimental capacity of the proprioceptive sensing area of the structure. The value of the resistance $R_{c r}=10 \mathrm{M} \Omega$ must be large enough so as not to disrupt the integration function of the circuit, but not so large so as to avoid the discharge current to pass through it. The choice also depends on the cutoff frequency of the system, which should stay low to avoid filtering of low frequencies.

\subsection{Experimental characterization of the deflection}

The vibrational dynamics are recorded experimentally by applying to the piezoactuator a low-amplitude sine input $U$ of increasing frequency. At low amplitude and relatively high frequencies, the creep and hysteretical behaviour of PZT can be neglected [13]. Using a spectrum analyser device (HP3562A), Bode diagram is recorded and the corresponding transfer is directly identified in Laplace domain (see figure 12). As expected by FlexIn optimization, the two first resonances modes are dominant over the following vibrational modes: this is the first point of interest in considering the criterion $J^{2,2,3}$ for the design. Let us also note that the pole/zero alternate pattern is kept into this desired spectrum of interest. Identification process is thus performed considering only these first two modes in a reduced model. For identification, we consider a second-order modal transfer expansion :

$$
G_{c o}(s)=\sum_{i=1}^{2} \frac{k_{c_{i}}}{1+\frac{2 \xi_{i}}{\omega_{n i}} s+\frac{1}{\omega_{n i}^{2}} s^{2}}=\frac{N_{c o}(s)}{D(s)}
$$

This transfer function is related to the state-space model according to the following relationship:

$$
G_{c o}(s)=C_{c o}(s I-A)^{-1} B
$$

Damping ratio $\xi_{1}=2.0 \%$ and $\xi_{2}=2.5 \%$ have been calculated from the measured quality factor at $-3 \mathrm{~dB}$ on the Bode diagram, according to $Q_{i}^{-3 d B} \approx \frac{1}{2 \xi_{i}}$, while values of the natural pulsations $\omega_{n 1}$ and $\omega_{n 2}$ are easily estimated owings to the measured resonance frequencies. Identified natural frequency values are reported in Table.1.

Table 1. Experimental and theoretical natural pulsations of $D$ transfer function.

\begin{tabular}{l|cc}
\hline \hline Natural frequency & $\omega_{n_{1}}(H z)$ & $\omega_{n_{2}}(H z)$ \\
\hline Theoretical FlexIn values & 2239.9 & 3800.1 \\
Experimental values & 2180.4 & 3517.3 \\
Relative errors & $2.66 \%$ & $7.44 \%$ \\
\hline \hline
\end{tabular}

Static gain $k_{c_{1}}$ and $k_{c_{2}}$ are computed to respect the frequency of the first antiresonance (between the two first resonances) and the whole system static gain. This 
identification procedure gives:

$$
\begin{aligned}
N_{c o}(s)= & 2.311 \times 10^{-9} s^{2}+2.22 \times 10^{-6} s+0.0502 \\
D(s)= & 8.837 \times 10^{-18} s^{4}+1.553 \times 10^{-14} s^{3}+ \\
& 6.802 \times 10^{-9} s^{2}+4.934 \times 10^{-6} s+1 .
\end{aligned}
$$

Identified response model $G_{c o}(s)$ is compared with the experimental behaviour in frequency (figure 12) and times (figure 13) domains.
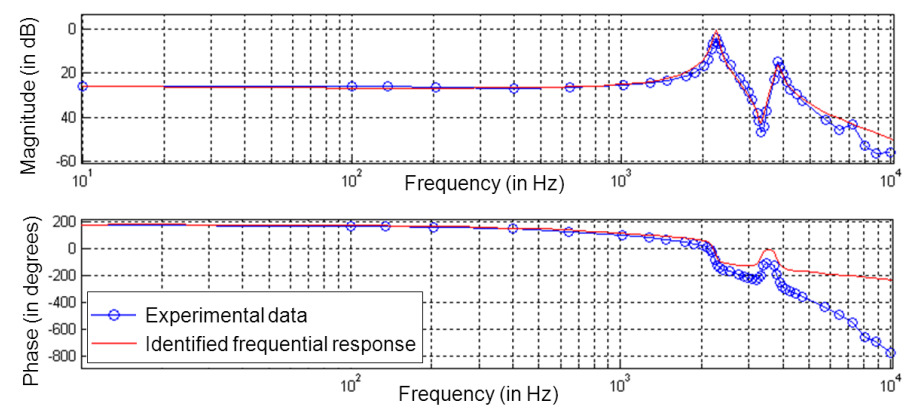

Figure 12. Experimental Bode diagram of the $\frac{\delta}{U}$ transfer $(\delta$ in $\mu \mathrm{m}$ and $U$ in $\mathrm{V})$ and corresponding identified transfer $G_{c o}$.

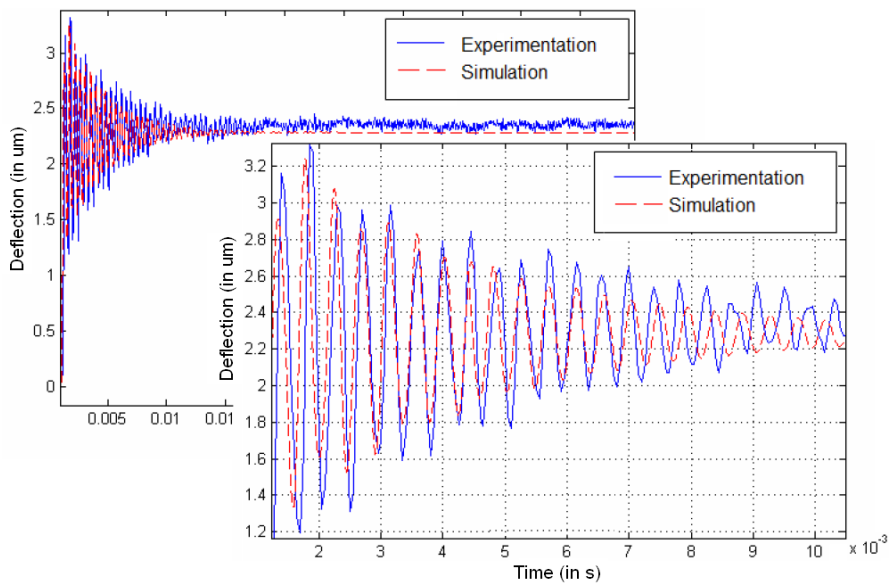

Figure 13. Experimental transient response for the $\frac{\delta}{U}$ transfer ( $\delta$ in $\mu \mathrm{m}$ and $U$ in $\mathrm{V}$ ) and corresponding identified transfer $G_{c o}$.

\subsection{Experimental characterization of the charge transfer}

The experimental protocol used for the identification of the piezoelectric sensing dynamics behaviour is similar to the one used for the identification of the deflection dynamics: the spectrum analyzer sends an excitation voltage to the actuation area of the smart structure, while the output of the electronic measurement circuit is recorded for the frequency response analysis. Measurements are reported in the figure 14. 
Taking into account the criterion $J^{k, n, v s}$, the optimisation design procedure of the system is effective: the modal observability by the charge transfer function $G_{o b}(s)$ of the first two dominant modes of the deflection transfer function $G_{c o}(s)$, is important (see the figure 15). The roll-off at higher frequencies allows to bring the identification of the $G_{o b}(s)$ transfer function to a model of order 4 , with a vibrational dynamics $D(s)$ similar to that corresponding to the actuation one:

$$
G_{o b}(s)=\sum_{i=1}^{2} \frac{k_{o_{i}}}{1+\frac{2 \xi_{i}}{\omega_{n i}} s+\frac{1}{\omega_{n i}^{2}} s^{2}}=\frac{N_{o b}(s)}{D(s)}
$$

or equivalently

$$
G_{o b}(s)=C_{o b}(s I-A)^{-1} B
$$
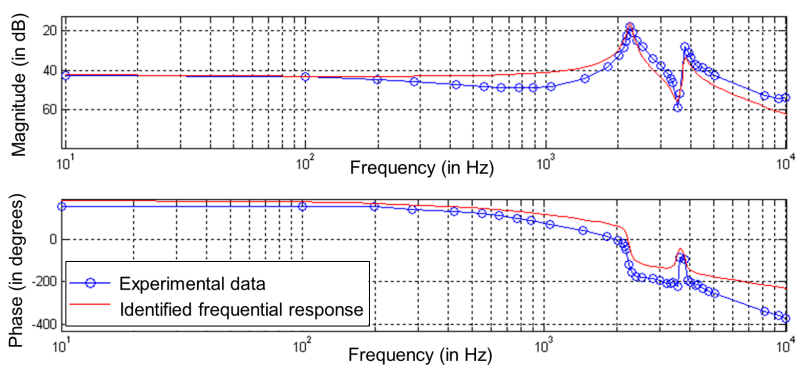

Figure 14. Experimental Bode diagram of the $\frac{q}{U}$ transfer function $(q$ in $\mathrm{C}$ and $U$ in V) and corresponding identified transfer function $G_{o b}$.

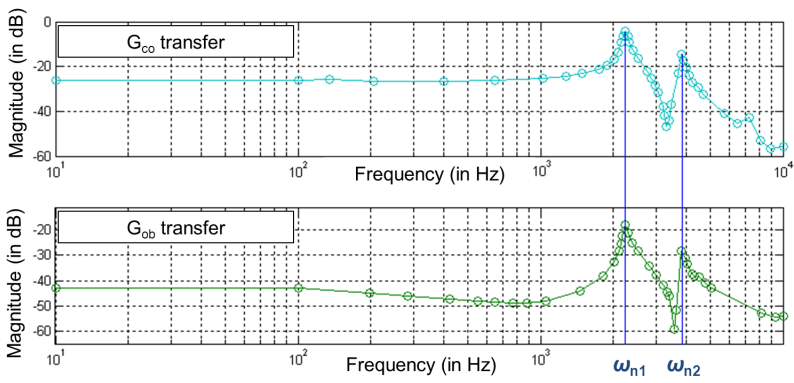

Figure 15. Correspondence of the dominant modes $\omega_{n_{1}}$ and $\omega_{n_{2}}$ in both deflection and charge transfer functions.

The computation of the static gain values $k_{o_{1}}$ and $k_{o_{2}}$ are based on the identification of the zero (antiresonance) of the experimental charge transfer function and static gain, so that :

$$
N_{o b}(s)=-1.407 \times 10^{-11} s^{2}-1.506 \times 10^{-8} s-0.007
$$

With the same damping ratio $\xi_{1}$ and $\xi_{2}$ as for the deflection transfer function $D(s)$ of $G_{c o}(s)$, the identified response model $G_{o b}(s)$ well matches the experimental response, in frequency (figure 14), and time (figure 16) domains. 


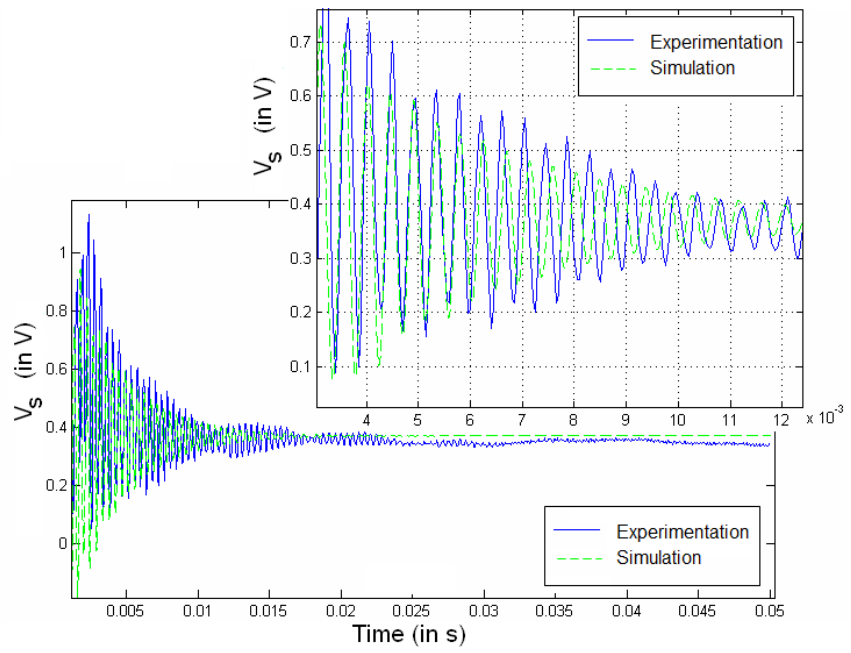

Figure 16. Experimental step response for the $\frac{V_{s}}{U}$ transfer function $\left(V_{s}\right.$ in $\mathrm{V}$ and $U$ in $\mathrm{V}$ ) and corresponding identified transfer function $G_{o b}$ with the electronic instrumentation.

The reduced model can then be used with confidence owings to the optimal criterion

$J^{k, n, t h v}$. The proposed optimization procedure is therefore relevant to design compliant structures that will be easily observable and controlable in an integrated way.

\section{Conclusions}

A new optimal design method for integrated smart compliant micromechatronic structure has been presented in this paper. In this method, an assembly of passive, active or sensitive building PZT blocks are optimally integrated in a monolithic structure. Actuation principle exploits the inverse piezoelectric effect, while the sensing principle is based on the direct effect. Complex multi-objective design problems can therefore be solved, taking into account many versatile criteria to synthesize high performance microrobotic flexible mechanisms. In addition to classical mechanical criteria (currently encountered in topology optimization: force and displacement maximization), the proposed software FlexIn can consider simultaneously control- and observation-based criteria to integrate the actuation and sensing capabilities in the structure in an optimal way.

From the static point of view, the proprioceptive sensing areas of the flexible structure are designed to maximize the measured electric charges. This objective satisfies requirements for a good working of electronic measuring instruments. At the same time, actuation areas are placed to maximize some mechanical criteria, such as the steadystate tip deflection. From the dynamic point of view, the optimal design ensures that dominant vibrational modes related to tip deflection are fully observable from the integrated sensing parts. This ensures a good integrated tip deflection reconstruction. These static and dynamic criteria allow the design of appropriate topologies for the compliant structure. These good properties will turn out to be essential in feedback or 
feedforward control of the device.

Based on the proposed method, an optimal micromechanism was designed and manufactured. The characteristics of this device were experimentally measured and compared to simulations and optimal design specifications. The results confirm the good properties of the prototype and the validity of this method for the optimal design of micromechatronic actuated devices.

\section{Appendix}

Matrices involved in the electromechanical model (1) and (2) of the beam displayed in the figure 3 :

$$
\begin{aligned}
& \mathbf{M}_{\mathbf{b}}=\rho A\left(\begin{array}{cccccc}
\frac{L}{3} & 0 & 0 & \frac{L}{6} & 0 & 0 \\
& \frac{13 L}{35} & \frac{11 L^{2}}{210} & 0 & \frac{9 L}{70} & -\frac{13 L^{2}}{420} \\
& & \frac{L^{3}}{105} & 0 & \frac{13 L^{2}}{420} & -\frac{L^{3}}{140} \\
& & & \frac{L}{3} & 0 & 0 \\
& (\text { sym. }) & & & \frac{13 L}{35} & -\frac{11 L^{2}}{210} \\
& & & & & \frac{L^{3}}{105}
\end{array}\right)_{R_{p}} \\
& \mathbf{K}_{\mathbf{b}}=Y\left(\begin{array}{cccccc}
\frac{A}{L} & 0 & 0 & -\frac{A}{L} & 0 & 0 \\
& \frac{12 I}{L^{3}} & \frac{6 I}{L^{2}} & 0 & -\frac{12 I}{L^{3}} & \frac{6 I}{L^{2}} \\
& & \frac{4 I}{L} & 0 & -\frac{6 I}{L^{2}} & \frac{2 I}{L} \\
& & & \frac{A}{L} & 0 & 0 \\
& \text { (sym.) } & & & \frac{12 I}{L^{3}} & -\frac{6 I}{L^{2}} \\
& & & & & \frac{4 I}{L}
\end{array}\right)_{R_{p}} \\
& \mathbf{G}_{\mathbf{b}}=Y h d_{31}\left(\begin{array}{cccccc}
1 & 0 & 0 & -1 & 0 & 0 \\
-1 & 0 & 0 & 1 & 0 & 0
\end{array}\right)_{R_{p}}^{t} \\
& \mathbf{C}_{\mathbf{b}}=\left(\begin{array}{cc}
\frac{h L \tilde{\varepsilon}_{33}}{e_{p}} & -\frac{h L \tilde{\varepsilon}_{33}}{e_{p}} \\
(\text { sym. }) & \frac{h L \tilde{\varepsilon}_{33}}{e_{p}}
\end{array}\right)_{R_{p}}^{t}
\end{aligned}
$$

$I=\frac{e_{p} h^{3}}{12}$ is the inertia moment of the beam cross section $A=e_{p} h, \rho$ is the density of the beam, $Y$ its Young's modulus defined as $Y=1 / s_{11}, \tilde{\varepsilon}_{33}=\varepsilon_{33}^{T}\left(1-k_{31}^{2}\right)$ is the modified electric permittivity and $k_{31}=\frac{d_{31}}{\sqrt{\varepsilon_{33}^{T} s_{11}^{E}}}$ its electromechanical factor coupling.

\section{References}

[1] Agnus J., Nectoux P., Chaillet N., "Overview of microgrippers and micromanipulation station based on a MMOC microgripper", Proceedings of the IEEE International Symposium on Computational Intelligence in Robotics and Automation, CIRA, Espoo, Finland, 2005, pp. 117123. 
[2] Breguet J.M., and al., "Monolithic piezoceramic flexible structure for micromanipulation", 9th International Precision Engineering Seminar and 4th International Conference on Ultraprecision in Manufacturing Engineering, pp. 397-400, Braunschweig, Germany, 1997.

[3] Janocha H., "Adaptronics and Smart Structures - Basics, Materials, Design and Applications", Second Edition Springer Berlin Heildelberg New-York, ISBN 978354071965 6, 2007.

[4] Leleu S., Abou-Kandil H., Bonnassieux Y., "Piezoelectric Actuators and Sensors Location for Active Control of Flexible Structures", IEEE Trans. on Instrumentation and Measurement, vol.50, No.6, pp.1577-1582, 2001.

[5] Moallem, M., Kermani M.R., Patel R.V., Ostojic M., "Control of a positioning system with structural flexibility using piezoelectric transducers", 42nd IEEE Conference on Decision and Control, vol. 5, pp. 4505-4510, 2003.

[6] Han J. H., Lee I., "Optimal placement of piezoelectric sensors and actuators for vibration control of composite plate using genetic algorithms", Smart Materials and Structures, vol. 8, pp 257-267, 1999.

[7] Silva E. C. N., Nishiwaki S., and Kikuchi N., "Design of piezocomposite materials and piezoelectric transducers using topology optimization - Part II," Computational Methods in Engineering, vol. 6, pp. 191-222, 1999.

[8] Wang S. Y., Tai K., Quek S. T., "Topology optimization of piezoelectric sensors/actuators for torsional vibration control of composite plates," Smart Materials and Structures, vol. 15, pp. 253-269, 2006.

[9] Maddisetty H., Frecker M., "Dynamic topology optimization of compliant mechanisms and piezoceramic actuators", ASME Journal of Mechanical Design, vol. 126, pp. 975-983, 2002.

[10] Juuti J., Kordas K., Lonnakko R., Moilanen V.-P., Leppavuori S., "Mechanically amplified large displacement piezoelectric actuators", Sensors and Actuators A: Physical, vol. 120, pp.225 - 231, 2005.

[11] Grossard M., Rotinat-Libersa C., Chaillet N., "Redesign of the MMOC microgripper piezoactuator using a new topological method", IEEE/ASME International Conference on Advanced Intelligent Mechatronics, Zurich, Switzerland, 2007.

[12] Grossard M., Rotinat-Libersa C., Chaillet N., Boukallel M., "Mechanical and control-oriented design of a monolithic piezoelectric microgripper using a new topological optimisation method", IEEE/ASME Transactions on Mechatronics, vol. 14, pp.32-45, 2009.

[13] Grossard, M., Boukallel M., Chaillet N., Rotinat-Libersa C. , "Modeling and Robust Control Strategy for a Control-Optimized Piezoelectric Microgripper", IEEE/ASME Transactions on Mechatronics, vol. 16, pp.674-683, 2010.

[14] Moussa R.E.K., Grossard, M., Chaillet N., Boukallel M., Hubert A., "Optimal design and control simulation of a monolithic piezoelectric microactuator with integrated sensor", IEEE/ASME International Conference on Advanced Intelligent Mechatronics (AIM), pp. 420-425, 2010.

[15] Grossard M., Rotinat-Libersa C., Chaillet N., "Gramian-based optimal design of a dynamic stroke amplifier compliant micro-mechanism", IEEE/RSJ International Conference on Robots and Systems, San Diego, USA, 2007.

[16] Preumont A., "Vibration control of active structures: an introduction, 2nd edition", Kluwer academic publishers, 2002.

[17] Hac A., Liu L., "Sensor and actuator location in motion control of flexible structures", Journal of Sound and Vibrations, vol. 167, pp. 239-261, 1993.

[18] Jonckheere E. A., "Principal components analysis of flexible systems - Open-loop case", IEEE Trans. on Automatic Control, vol. 29, pp. 1095-1097, 1984.

[19] Gawronski W., Lim K. B., "Balanced control of Flexible structures", Springer, London, 1996.

[20] PI Piezo Ceramic Technology, 2005. Available: http://www.piceramic.com/. 